\title{
Infecciones por Vibrio parahaemolyticus e intoxicaciones por algas: problemas emergentes de salud pública en Chile
}

\author{
Cristina Hernández $G^{1, a}$, Juanita U lloa $P^{2, b}$, José Antonio \\ Vergara $0^{2}$, Romilio Espejo $\mathrm{T}^{3, a}$, Felipe Cabello $\mathrm{C}^{4}$. \\ Vibrio parahaemolyticus infections \\ and algal intoxications as emergent \\ public health problems in Chile
}

There is interest in the paradigm that relates environmental sea changes to the emergence of diseases that affect both aquatic organisms in the sea and human beings. The emergence of Vibrio parahaemolyticus as an important cause of epidemic summer diarrhea in 2004 and 2005, confined mainly to the tenth region in Chile, could be a manifestation of this trend. This and other areas of the country have also experienced several outbreaks of paralytic shellfish poisoning (PSP), diarrheal shellfish poisoning (DSP) and amnesic shellfish poisoning (ASP) caused by harmful algal blooms (HAB) of Alexandrium catenella, Dinophysis acuta and Pseudonitzchia species, respectively. The short historical record of these pathological phenomena in Chile suggests that they are increasing in frequency and expanding their geographical range. The $\mathrm{V}$ parahaemolyticus isolates responsible for the Chilean outbreaks correspond mainly to the pandemic strain O3:K6. HAB found in Chile and the intoxications caused by them have similar biological characteristics to those described in other areas of the world. The tenth region, the area where these problems are emerging, produces approximately $80-90 \%$ of the shellfish consumed in Chile and a large proportion of the shellfish that is exported. Prevention of these public health problems can be attained by developing policies that increase environmental surveillance for Vibrios and toxic algae, improve the epidemiological surveillance of acute diarrhea and algal intoxications after the ingestion of raw bivalves, and educate the population on the mode of transmission of these diseases. Scientific capacity and laboratories need to be developed to widen the limited knowledge of the biology of Vibrio and toxic algae and the environmental factors that favor their emergence as public health and economic problems in Chile (Rev Méd Chile 2005; 133: 1081-88).

(Key Words: Bacterial proteins; Diarrhea; Paralytic shellfish poisoning; Vibrio parahaemolyticus)
Recibido el 27 mayo, 2005. Aceptado el 27 julio, 2005.
${ }^{1}$ Laboratorio de Bromatología, Autoridad Sanitaria, Secretaría Regional Ministerial de Salud X Región. O’Higgins 276, Puerto Montt, X Región, Chile. ²Unidad de Epidemiología, Auto- ridad Sanitaria Secretaría Regional Ministerial de Salud X Región. Seminario 359, Puerto Montt, X Región, Chile. ${ }^{3}$ Instituto de Nutrición y Tecnología de los Alimentos, Universidad de Chile. El Líbano 5524, Macul, Santiago, Chile. ${ }^{4}$ Department of Microbiology and Immunology, New York Medical College, Valhalla, NY 10595, EE.UU.
aBioquímico
${ }^{\mathrm{b}}$ Enfermera Universitaria

Correspondencia a: Dr. Felipe C Cabello. Department of Microbiology and Microbiology, New York Medical College, Valhalla, NY 10595, USA. Teléfono: +914-594-4182. Fax: +914-594-4176. E mail: cabello@nymc.edu 
$E^{\mathrm{a}}$ los últimos años, a las enfermedades clásicas transmitidas a través del consumo de peces y mariscos y por el contacto e ingestión de agua de mar, tales como cólera, fiebre tifoidea y hepatitis $\mathrm{A}^{1}$, se han agregado una serie de nuevos problemas de enfermedades infecciosas y toxi-infecciosas que tienen este mismo modo de transmisión ${ }^{2-7}$. Sin embargo, los patógenos responsables de estos nuevos procesos son autóctonos del nicho ecológico marino ${ }^{2,3,8-10}$, no se originan por contaminación de este nicho con materia fecal $2,3,5,8-10$ y el hospedero humano no está envuelto en sus ciclos biológi$\cos ^{1-3,5,8-11}$, por lo que el acceso de estos agentes a este hospedero se produce de forma acciden$\mathrm{tal}^{2,3,5,8-10}$. Una característica de este nuevo grupo de enfermedades es que, dada su reciente aparición, la biología de los patógenos que la producen, las causas de su aparición y su epidemiología están en gran medida indefinidas. Sin embargo, ellos tienen una gran relevancia, ya que pueden producir un alto número de episodios de morbilidad, casos de mortalidad y grandes pérdidas económicas y alteraciones sociales en la población dedicada a industrias extractivas y procesadoras de productos del $\mathrm{mar}^{2,3}$. En el caso de Chile, la aparición de estas nuevas patologías se ha manifestado por epidemias de diamea aguda producidas por Vibrio parahaemolyticus, en varias regiones del país ${ }^{12,13}$. Similarmente, en los últimos tiempos, ha habido en Chile un aumento substancial de floración de algas nocivas (FANs), que se han acompañado de intoxicaciones humanas, secundaria a la ingesta de mariscos bivalvos, contaminados con las toxinas producidas por estas algas ${ }^{14-16}$. La creciente magnitud de estos fenómenos ${ }^{13,14,16}$, indicarían que se están convirtiendo en un importante problema de salud pública en Chile ${ }^{13-16}$. El objeto de esta revisión es introducir al lector de la revista a este tema de gran interés científico y sanitario.

\section{INFECCIONES POR VIBRIO PARAHAEMOLYTICUS}

Hábitat, vectores y modo de transmisión. El V parahaemolyticus es una bacteria halófila Gram negativa, que al igual que múltiples otros vibrios marinos, es una de las especies que compone la flora nomal en estuarios y del agua de mar y de algunos organismos que en ella viven ${ }^{5,17-19}$. El número de $\mathrm{V}$ parahaemolyticus en el agua de mar estańa asociado, al igual que el Vibrio cholerae ${ }^{20,21}$, a la concentración de zoo- plancton, especialmente a copépodos y a la temperatura ${ }^{20,21}$. Esto indicańa que las concentraciones de $\mathrm{V}$ parahaemolyticus en el agua de mar pueden variar con aquellos factores que produzcan variaciones del zooplancton, incluyendo temperatura, luminosidad, comientes marinas, concentración de nutrientes y concentración del fitoplancton, entre muchos otros ${ }^{19-}$ 25. El V parahaemolyticus, por estar en suspensión en el agua de mar, se concentra en moluscos filtradores bivalvos que en nuestro país son una importante parte de la dieta diaria de la población, incluyendo choros, Choromytilus choro, choritos, Mytilus chilensis, cholgas, Aulacomya ater, navajuelas, Tangelus dombeii, machas, Mesoderma demacium, almejas, Venus antiqua, culengues, Gari solida, y ostiones, Argopecten purpuratus, en cuyas carnes puede alcanzar grandes concentraciones 5,6,10,26. Además, en otros países, esta bacteria se ha encontrado contaminando camarones y came de cangrejo y de jaiva $5,6,10,26 \mathrm{y}$, ocasionalmente, came de pescado ${ }^{5,6,10,26}$. La infección humana se adquiere por la ingestión de estos productos del mar, ya sea crudos o parcialmente cocidos, conteniendo concentraciones infecciosas de la bacteria, que algunos estudios han caracterizado como de 1 x $10^{4}$ bacteria por gramo de maris$\mathrm{co}^{5,6,10,24,26,27}$. Factores importantes en la concentración de $\mathrm{V}$ parahaemolyticus, en las cames de bivalvos ingerida son la concentración inicial de la bacteria, la temperatura de almacenamiento y de transporte, y la temperatura de cocción ${ }^{5,6,10,19,24,26-29}$. V parahaemolyticus tiene un tiempo de generación a la temperatura de $37^{\circ}$ de 8 a $9 \mathrm{~min}$, de tal modo que puede alcanzar concentraciones muy altas en poco tiempo, ya que la bacteria puede crecer después de la recolección de los mariscos si la temperatura es la adecuada para este crecimiento 5,6,10,18,19,22,26-29.

Microbiología y virulencia. La especie V parahaemolyticus tienen una gran diversidad y sus miembros pueden diferir en hasta $60 \%$ en la secuencia del ADN de sus genomas ${ }^{30,31}$. Esta diversidad es bastante clara en los V parahaemolyticus ambientales aislados del agua de mar, de mariscos bivalvos y de peces ${ }^{13,19}$. Sin embargo en las epidemias recientes, esta diversidad es indetectable en aquellos vibrios aislados de las deposiciones de pacientes con infecciones producidas por esta bacteria ${ }^{5,6,13,19}$. De esta manera, los vibrios patogénicos parecen representar un reducido segmento de esta diversidad y se caractenzan, en general, por tener características fenotípicas y genotí- 
picas bien definidas 5,6,13,19. El V parahaemolyticus causante de epidemias en Antofagasta en 1998 y en la X región de Chile en 2004 (Figura 1) y 2005, tiene los antígenos O3:K6, presenta el fenómeno de Kanagawa y es ureasa negativo, Tabla 12,13. Además, tiene los genes asociados a la virulencia tdh and toxRS/new ${ }^{13}$, y estudios moleculares de su genoma han demostrado que pertenece al clon patogénico que emergiera en el sudeste asiático en 1996, el cual se ha diseminado prácticamente por el todo el mundo (Tabla 1) $)^{5,13,19}$. Los factores de virulencia de estos vibrios patogénicos están poco caracterizados e incluyen los productos de los genes tdh, que codificanía por una hemolisina termoestable asociada al fenómeno de Kanagawa ${ }^{5,13,19}$ y el gene trh, que codificańa por una hemolisina relacionada a la hemolisina termo estable $5,13,19$. La habilidad de producir ureasa, captar hierm, y enzimas extracelulares con capacidad hemolítica como lipasas, también han sido asociada a la patogenicidad de vibrio, pero los mecanismos por el cual estas actividades aumentan la virulencia no han sido caracterizados $5,37,38$. Sin embargo, no existe duda que el cuadro diameico es producido por la infección y la producción de factores de virulencia a nivel local del intestino $0^{5,19,37}$. De tal forma que, la inactivación del vibrio por el calor u otros tratamientos en los alimentos que lo contienen previene la infección ${ }^{5,10,19,37 . ~}$

Enfermedad, tratamiento y prevención. La enfermedad producida por $\mathrm{V}$ parahaemolyticus es una diamea aguda, generalmente de tipo secretorio y que en algunas ocasiones puede ser hemorrágica ${ }^{26,39}$, generalmente acompañada de cólicos, náuseas, a veces vómitos y fiebre ${ }^{26,39}$. Esta gastroenteritis tiene un período de incubación de 12 a 48 h y, en general, se resuelve de manera espontánea en dos 0 tres días ${ }^{26,39}$. En personas con diabetes, enfermedad hepática e inmunosupresión, la enfermedad puede ser severa y acompañarse de bacteremia 26,39 . El V parahaemolyticus puede también infectar heridas, $\mathrm{y}$ desde allí puede también diseminarse y producir bacteremia fulminante con hipotensión ${ }^{26,39}$. Con el antecedente de la ingesta reciente de mariscos bivalvos crudos o parcialmente cocidos, el diagnóstico clínico de diarrea aguda producido por Vibrio para-

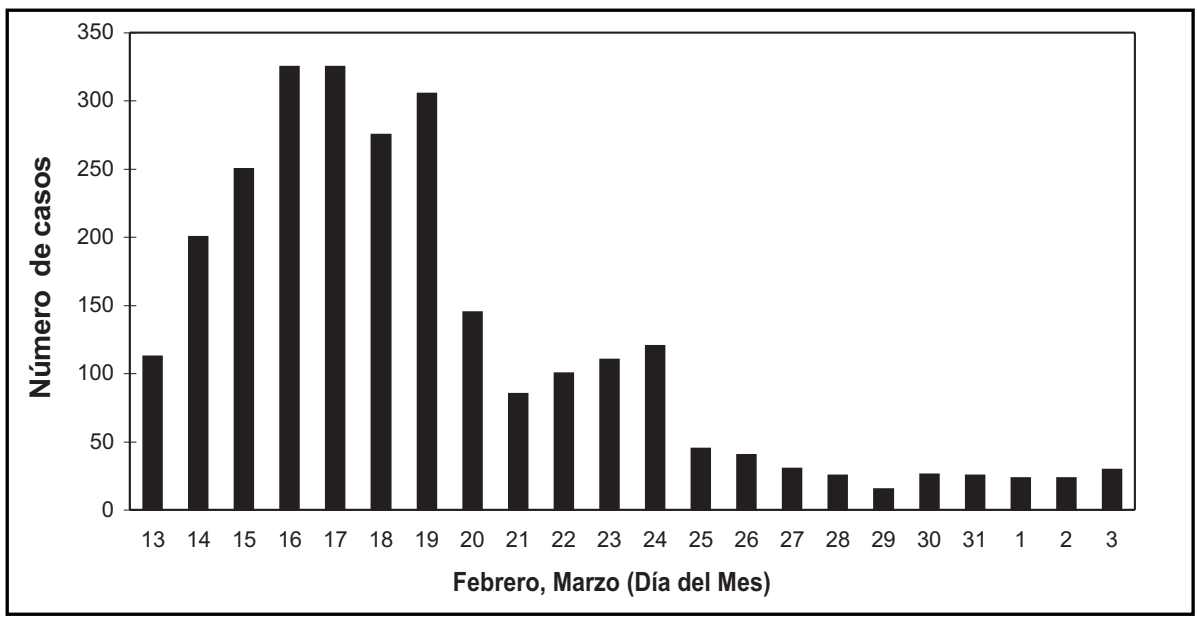

Figura 1. Epidemia de diarrea por Vibrio parahaemolyticus en X Región, 2004.

Tabla 1. Características de las cepas de Vibrio parahaemolyticus aisladas de casos clínicos in Chile

\begin{tabular}{|lcccccc|}
\hline & Serotipo & tdh & trh & Ureasa & Ka & toxRS/new \\
\hline Antofagasta, 1998 & 03:K6 & + & - & & + & + \\
Puerto Montt, 2004 & 03:K6 & + & - & - & + & + \\
\hline
\end{tabular}

Fuente: González-Escalona et al, 2005. Emerg Infect Dis 11: 29-131.

Significado de las abreviaciones: tdh: gene de la hemolisina directa termoestable (thermostable direct haemolysin); trh: gene de la hemolisina relacionada con la hemolisina termoestable (tdh-related haemolysin); Ka: fenómeno de Kanagawa; toxRS/new: nuevos genes toxigénicos RS. 
haemolyticus es relativamente fácil de hacer y es complementado por la detección de esta bacteria en el coprocultivo sembrado en placas de TCBS (tiosulfate-citrato-sales biliares-sucrosa) previo enriquecimiento en agua peptonada alcalina ${ }^{40,41}$. Reacciones bioquímicas ayudan a diferenciar entre $\mathrm{V}$ parahaemolyticus y otros vibrios productores de patología humana como Vibrio cholerae, Vibrio vuinificus y Vibrio mimicus ${ }^{40,42}$. La prevención de las infecciones por V parahaemolyticus, en Estados Unidos de Norteamérica (EEUU) y Canadá, se basa principalmente en el manejo de áreas de extracción debido a que los mariscos tienen la certificación de origen y, por otro lado, existe vigilancia epidemiológica constante que detecta precozmente la aparición de casos de diarrea aguda después de la ingestión de maniscos crudos $7,17,24,27$. Una vez que los casos de diarrea aguda se han detectado, la otra medida del manejo preventivo de estas infecciones es el cieme de las áreas de recolección de mariscos bivalvos, cuando, durante monitoreos continuos se detecta un aumento del número de bacterias por gramo de manisco crudo y éste llega a 1 x $10^{4}$ 27,35,41,47,48. Sin embargo, no está claro si en Chile estos mismos parámetros son aplicables ya que en la carne los mariscos chilenos el V parahaemolyticus no alcanza las concentraciones a que llega en otros países (Hemández C. Datos no publicados). La educación del público respecto de los peligros de la ingesta de maniscos crudos es también una importante medida de prevención $7,17,24,27$.

\section{INTOXICACIONES POR FLORACIÓN DE ALGAS NOCIVAS} (FANs)

FANs. Las intoxicaciones humanas como resultado de FANs en el mar se han presentado en diversas partes del mundo y es una patología que pareciera estar en indiscutible aumento ${ }^{2-4,42-45}$. Las neurotoxinas y otras toxinas producidas por las algas que componen estos FANs, que generalmente son dinoflagelados o diatomeas, son adquiridas por mariscos, zooplancton 0 peces herbívoros, que se alimentan mediante la filtración del agua de mar, que contiene a estas algas en suspensión ${ }^{2-4,42-45}$. Los mariscos bivalvos, como los mencionados precedentemente, concentran en su came a estas toxinas y actúan como vectores directos de ellas ${ }^{2-4,42-45}$, y los síntomas tóxicos se manifiestan después de la ingestión de éstos, ya sean crudos o cocidos, ya que las toxinas de este origen son termomesistentes ${ }^{2-4,42-45}$. Sin embargo, en algunas condiciones, el zooplancton y los peces herbívoros pueden transmitir las toxinas a organismos superiores dentro de la cadena trófica y éstos, a su vez, transmitir las toxinas directamente a humanos ${ }^{4,46}$. La capacidad de los mariscos bivalvos para concentrar estas toxinas se debe a que son capaces, en promedio, de filtrar 30 litros de agua al día,42-45. Los tipos de intoxicaciones producidas por mariscos están señalados en la Tabla 2 y en ella también se señalan los tipos de intoxicaciones por mariscos presentes en Chile y la fecha de su detección en el país ${ }^{4,47}$. La misma Tabla 2 señala las

Tabla 2. Síndromes tóxicos asociados a toxinas de algas marinas

\begin{tabular}{|c|c|c|c|c|c|}
\hline Síndrome & Organismo causal & Vector & Toxina & Blanco & Presencia en Chile \\
\hline VPM & $\begin{array}{l}\text { Alexandrium spp. } \\
\text { Gymnodinium spp. } \\
\text { Pyrodinium spp. }\end{array}$ & Mariscos & Saxitoxinas & Canales de sodio, Sitio 1 & $\begin{array}{l}\text { X, XI y XII } \\
\text { Regiones desde el } \\
\text { año } 1970\end{array}$ \\
\hline VDM & $\begin{array}{l}\text { Dinophysis spp. } \\
\text { Prorocentrum spp. }\end{array}$ & Mariscos & $\begin{array}{l}\text { Dinophysistoxinas } \\
\text { Acido okadaico }\end{array}$ & Fosfatasa serina/treonina & $\begin{array}{l}\text { X Región desde el } \\
\text { año } 1970\end{array}$ \\
\hline VAM & Pseudo-nitzschia spp. & Mariscos & Acido domoico & Receptores de glutamato & $\begin{array}{l}\text { IV y X Regiones } \\
\text { desde el año } 2000\end{array}$ \\
\hline VC & Gambierdiscus toxicus & Peces de corales & Ciguatoxinas & $\begin{array}{l}\text { Canales de sodio, } \\
\text { Sitio } 5\end{array}$ & No \\
\hline VNM & Gymnodinium breve & Mariscos & Brevetoxinas & $\begin{array}{l}\text { Canales de sodio } \\
\text { Sitio } 5\end{array}$ & No \\
\hline $\begin{array}{l}\text { Síndrome } \\
\text { del estuario }\end{array}$ & Pfiestenia piscicida & Agua & Desconocida & Desconocido & No \\
\hline
\end{tabular}

Fuentes: Van Dolah FM 2000. Environ. Health Perspect. 108:Suppl. 1:133-141; Hernández, C. 2005. Datos no publicados. Significado de las abreviaciones: VPM, veneno paralítico de mariscos; VDM, veneno diarreico de mariscos; VAM, veneno amnésico de mariscos; $\mathrm{VC}$, veneno de ciguatera; VNM, veneno neurotóxico de mariscos. 
caractenísticas químicas de las neurotoxinas y de sus blancos ${ }^{4,47}$. También existen toxinas producidas por algas que pueden transmitirse a humanos por aerosoles como las brevetoxinas y volatilizadas como las toxinas producidas por algas del grupo Pfiesteria ${ }^{47}$.

FANs en Chile. La Tabla 2 muestra que en el año 1970 se detectaron por primera vez en Chile intoxicaciones por el veneno paralítico de mariscos (VPM) que sería el resultado de FANs de Alexandrium catenella en la región de Magallanes y que desde esa fecha se han extendido hacia el norte, hasta llegar a la latitud del canal Dalcahue, en la provincia de Chiloé, $X$ región ${ }^{14,16}$. El VPM ha producido en el país desde esa fecha aproximadamente 400 intoxicaciones con 26 fallecimientos ${ }^{14,16}$. En la X región, y aproximadamente desde el año 1972, se han producido varios brotes de diarrea aguda producido por el veneno diarréico de mariscos (VDM) que es producida por FANs de algas del grupo Dynophysis, y que han estado limitados al estuario del Reloncaví siendo el agente, en todos estos eventos, Dynophysis acuta (Hernández C. Datos no publicados). Sin embargo, en el evento de febrero y marzo de 2005 en la misma zona, aparece Dynophysis acuminata como agente productor de ácido okadaico (Hernández C. Datos no publicados). En Chile también se han detectado FANs producidos por diatomeas del género Pseudo-nitzchia que producen el veneno amnésico de mariscos (VAM) en varias regiones del país, incluyendo la $\mathrm{X}$ región, donde el año 2000 se produjo un florecimiento que afectó las zonas de Calbuco, Castro y Dalcahue y se alcanzaron valores de $260 \mu \mathrm{g}$ ácido domoico por gramo de marisco (Hernández C. Datos no publicados). Otros hallazgos de VAM se han producido en el norte en Bahía Inglesa, III región y La Serena ${ }^{14,16}$.

Cuadro clínico, tratamiento y prevención. La intoxicación por VPM se caracteriza por un período corto de incubación de menos de una hora y se manifiesta con parestesias y adormecimiento del área perioral y de las extremidades, con parálisis, incoherencia, disfagia y finalmente, puede causar muerte por parálisis respiratoria ${ }^{4,45,47,48}$. Esta sintomatología neurológica se resuelve espontáneamente en, aproximadamente, $12 \mathrm{~h}$ y la recuperación es la regla si no ha habido parálisis respiratoria ${ }^{4,45,47,48}$. La mortalidad está en relación directa con la dosis de VPM consumida ${ }^{4,45,47,48}$. La toxinas del grupo VDM produce diarrea leve con un tiempo de incubación corto, incluso de $30 \mathrm{~min}$, que se acompaña de vómitos $\mathrm{y}$, generalmente, se resuelve en dos a tres días y carece de complicaciones neurológicas ${ }^{4,45,47,48}$. El diagnóstico diferencial de la diarrea producida por el VDM se hace con diarreas bacterianas también trasmitidas por mariscos y pescados, como la producidas por $\mathrm{V}$ parahaemolyticus y Salmonella 4,47,48. Dentro del grupo de toxinas VDM, se encuentra el ácido okadaico, que ha sido identificado también como un promotor y supresor del desarrollo de tumores en animales $4,53,57$, sugiriendo que la ingesta crónica de ellos, a niveles no diarreicos, pudiera provocar cáncer, a través de alteraciones, en el ciclo celular, generadas por la intoxicación de las fosfatasas Ser/ Treo ${ }^{49,50}$. En este grupo también se encuentran toxinas alternativas como las dinofisistoxinas, yessotoxinas y pectenotoxinas, careciendo todas ellas de un efecto diarreico $0^{4,47,48}$.

El ácido demoico, VAM, produce síntomas gastrointestinales incluyendo diarrea después de su ingestión y ésta se puede acompañar de mareos, somnolencia, convulsiones, coma y pérdida de la memoria de eventos recientes ${ }^{4,47,48}$. La pérdida de la memoria y las alteraciones de conciencia pueden ser permanentes, ya que, especialmente en personas de edad, la toxina puede destruir el tejido nervioso responsable de estas funciones ${ }^{4,47,48}$, pudiendo estas complicaciones simular una demencia ${ }^{4,47,48}$. El tratamiento de estas intoxicaciones es sintomático, y en el caso de la intoxicación por VPM y VAM, la parálisis respiratoria y el coma pueden indicar el uso de respiración asistida 4,47,48. Similarmente a lo que sucede con las infecciones por $\mathrm{V}$ parahaemolyticus, la presencia del cuadro clínico con el dato de la ingesta de mariscos bivalvos, en este caso crudos o cocidos, y a veces pescado, es crucial para el diagnóstico de esta patología ${ }^{4,44-48}$. La prevención de estas intoxicaciones está basada en la vigilancia ambiental respecto de la presencia de estas diferentes toxinas en mariscos y en la prohibición de extracción y comencialización si éstos están contaminados con ellas ${ }^{4,44-48}$. La detección en el ambiente marino de las algas que las producen a través de monitoreos de fitoplancton, la pesquisa temprana de casos clínicos de intoxicaciones debidos a ellas, y la educación de la 
población respecto de la presencia de este problema y de los métodos de prevención son también útiles en esta tarea ${ }^{14,16,44-48}$.

\section{CONCLUSIONES}

Si bien es cierto que estas enfermedades son sólo ocacionalmente severas y son, en general, de recuperación espontánea, el brusco aumento de ellas, como ha sucedido durante el comienzo del año 2004 y de 2005 con las epidemias de V parahaemolyticus, puede sobrepasar la capacidad de los servicios asistenciales y comenzar a producir algunos casos de mortalidad por ineficiencias en la atención médica y porque el gran número de casos comienza a incluir a individuos en edades extremas y con patología concomitante, como diabetes, cirrosis, insuficiencia cardíaca e inmunodepresión ${ }^{4,39}$. En el año 2004, también se detectó, en el ambiente marino de la $\mathrm{X}$ región, la presencia de Vibrio vulnificus, lo que complica el escenario, debido a la mayor virulencia de este vibrio, que puede producir enfermedad severa en los pacientes con riesgo (ancianos, niños, cirróticos e inmunosuprimidos), y que tiene presenta-

\section{REFERENCIAS}

1. Foster EM. Historical overview of key issues in food safety. Emerg Infect Dis 1997; 3: 481-2.

2. ANDERSON D. Harmful algal blooms (HABs) in the United States. WHOI Sea Grant. Sea Grant Woods Hole Focal Points. WHOI-G-98-2004 C2. Sea Grant Program, Woods Hole Oceanographic Institution, MS\#2, Woods Hole, MA 02543, 1998.

3. ANDERSON D. The growing problem of harmful algae. Oceanus 2004; 43(2).

4. BRETT MM. Food poisoning associated with biotoxins in fish and shellfish. Curr Opin Infect Dis 2003; 16: 461-5.

5. Daniels NA, Evans MC, Griffin PM. Noncholera Vibrios. In W. M. Scheld, W. A. Craig, and J. M. Hughes (eds.), Emerging Infections 4. ASM Press, Washington, DC, 2000; 237-47.

6. Daniels NA, Ray B, Easton A, Marano N, Kahn E, MC Shan L ET aL. Emergence of a new Vibrio ciones clínicas como infecciones de herida y septicemia similares a las de Vibrio paraha emolyticus, con una letalidad de 50\% (Hernández C. Datos no publicados) $4,40,51$. Además, si los cambios ambientales ${ }^{52-54}$ que han generado la emergencia de esta patología persisten, cambios genéticos en $\mathrm{V}$ parahaemolyticus puede generar variantes más patogénicas capaces de producir enfermedad más severa ${ }^{13,18,19}$, pueden hacerse más comunes las infecciones con vibrios patógenos alternativos en humanos como V vulnificus ${ }^{19,51}$, y pueden generarse FANs con algas de toxicidad desconocida hasta ahora en el país ${ }^{14-}$ 16,55 .

En resumen, la aparición de este nuevo grupo de enfermedades debiera estimular a las autoridades sanitarias, a la comunidad científica, y a los organismos gubernamentales pesqueros y económicos a elaborar planes de gestión y contingencia con los adecuados presupuestos para enfrentar estos fenómenos sanitarios y ecológicos emergentes. Estos problemas de salud pública tienen el potencial de extenderse al resto del país, de afectar la salud de grandes grupos y de comprometer la salud económica de actividades como la pesca y la acuicultura y el prestigio del país como exportador de alimentos de buena calidad.

parahaemolyticus serotype in raw oysters: A prevention quandary. JAMA 2000; 84: 1541-5.

7. Potasman I, Paz A, Odeh M. Infectious outbreaks associated with bivalve shellfish consumption: a worldwide perspective. Clin Infect Dis 2002; 35: 921-8.

8. Altekruse SF, Bishop RD, Baldy LM, Thompson SA, WiLSON BJ, RAY BJ, GrifFin PM. 2000. Vibrio gastroenteritis in the US Gulf of Mexico region: the role of raw oysters. Epidemiol Infect 2000; 124: 489-95.

9. Burke DD, Committee on Climate Ecosystems, Infectious Disease and Human Health. Under the weather. Climate, Ecosystems, and Infectious Disease, National Academy Press, 2101 Constitution Ave., N.W., Washington, DC 20418, 2005; 1-44.

10. Daniels NA, Mac Kinnon L, Bishop R, Altekruse S, Ray B, Hammond RM et al. Vibrio parahaemolyticus infections in the United States, 1973-1998. J Infect Dis 2000; 181: 1661-6. 
11. Cangelosi GA, Freitag NE, Buckiey MR. From Outside to Inside: Environmental Microorganisms as Human Pathogens, American Academy of Microbiology, Washington, DC, 2004: 1-6.

12. Cordova JL, Astorga J, Silva W, Ruquelme C. Characterization by PCR of Vibrio parahaemolyticus isolates collected during the 1997-1998 Chilean outbreak. Biol Res 2002; 35: 433-40.

13. González-Escalona N, Cachicas V, Acevedo C, Rioseco ML, Vergara Ja, Cabello F et al. Vibrio parahaemolyticus diarrhea, Chile, 1998 and 2004. Emerg Infect Dis 2005; 11: 129-31.

14. Guzmán L, Pacheco H, Pizarro G, Alarcón C. Capítulo 11: Alexandrium catenella y veneno paralizante de los mariscos en Chile, In: E. A. Sar, M. E. Ferrario, and B. Reguera (eds.), Floraciones Algales Nocivas en el Cono Sur Americano. Instituto Español de Oceanografía, Madrid, España 2002; 235-56.

15. Molnet C, Lafon A, Lembey G, Moreno CA. Patrones de distribución espacial y temporal de floraciones de Alexandrium catenella (Whedon \& Kofoid) Balech 1985, en aguas interiores de la Patagonia noroccidental de Chile. Rev Chil Hist Nat 2003; 76: 681-98.

16. Suárez Ista BA, López A, Hernández C, Ciement A, GuzMÁn L Capítulo 12: Impacto económico de las floraciones de microalgas nocivas en Chile y datos recientes sobre la ocurrencia de veneno amnésico de los mariscos. En Sar EA, Ferrairo ME, Reguera B. (eds.), Floraciones Algales Nocivas en el ConoSur Americano. Instituto Español de Oceanografía, Mos, Pontevedra - Espana 2002; 257-68.

17. Lake R, Hudson A, CRessey P. Risk profile: Vibrio parahaemolyticus in seafood. Crown Research Institute. Institute of Environmental Science and Research Limited Christchurch Science Centre, P. O. Box 29 181, Christchurch, New Zealand, 2003.

18. MorRIS JG JR. Cholera and other types of Vibriosis: A story of human pandemics and oysters on the half shelf. Clin Infect Dis 2003; 37: 272-80.

19. Olver JD, Kaper JB. Vibrio species. In: Food Microbiology Fundamentals and Frontiers. (MP Doyle, LR Beuchat, TJ Montville, eds) ASM Press, Washington, DC, 1997; 228-64.

20. Lipp EK, Hug A, Colwell RR. Effects of global climate on infectious disease: the cholera model. Clin Microbiol Rev 2002; 15: 757-70.

21. LipP EK, RIverA ING, Gil AI, Espeiand EM, Choopun N, LouIS VR ET AL. Direct detection of Vibrio cholerae and ctxA in Peruvian coastal water and plankton by PCR. Appl Environ Microbiol 2003; 69: 3676-80.
22. De Paola A, Nordstrom L, Bowers JC, Wells JG, Cоок DW. Seasonal abundance of total and pathogenic Vibrio parahaemolyticus in Alabama oysters. Appl Environ Microbiol 2003; 69: 1521-6.

23. Kaneko T, Colwell RR. Ecology of Vibrio parahaemolyticus in Chesapeake Bay. J Bacteriol 1973; 113: 24-32.

24. Vibrio Parahaemolyticus Risk Assessment Task Force. Center for food safety and applied nutrition. Food and Drug Administration. U.S. Department of Health and Human Services. Draft Risk Assessment on the Public Health Impact of Vibrio parahaemolyticus in Raw Molluscan Shellfish 2000.

25. WatKINS WD, CABEL VJ. Effect of fecal pollution on Vibrio parahaemolyticus densities in an estuarine environment. Appl Environ Microbiol 1985; 49: 307-13.

26. Daniels NA, Shafaie A. A review of pathogenic of Vibrio infections for clinicians. Infect Med 2000; 17: 665-85.

27. De Paola A, Kaysner CA, Bowers J, Cook DW. Environment investigations of Vibrio parahaemolyticus in oysters after outbreaks in Washington, Texas, and New York (1997 and 1998). Appl Environ Microbiol 2000; 66: 4649-54.

28. Canadian Shelfish Quilty Resource. Vibrio parahaemolyticus. BCSGA Vp Control Program.

29. IsAacson RE, ToRrence M, Buckiey MR. Preharvest Food Safety and Security, American Academy of Microbiology, Washington, DC 2003: 1-16.

30. Makino K, Oshima K, Kurokawa K, Yokoyama K, Uda T, TAGomori $K$ ET aL. Genome sequence of vibrio parahaemolyticus: a pathogenic mechanism distinct from that of V cholerae. Lancet 2003; 361: 743-9.

31. Moreno C, Romero J, Espejo RT. Polymorphism in repeated 16S rRNA genes is a common property of type strains and environmental isolates of the genus Vibrio. Microbiology 2002; 148: 1233-9.

32. Chiou CS, Hsu SY, Chiu SI, Wang TK, Chao CS. Vibrio parahaemolyticus serovar O3:K6 as cause of unusually high incidence of food-borne disease outbreaks in Taiwan from 1996 to 1999. J Clin Microbiol 2000; 38: 4621-5.

33. Chowdhuny A, Ishibashi M, Thiem VD, Tuyet DT, Tung TV, CHIEN BT ET AL. Emergence and serovar transition of Vibrio parahaemolyticus pandemic strains isolated during a dianhea outbreak in Vietnam between 1997 and 1999. Microbiol Immunol 2004; 48: 319-27.

34. Chowdhury NR, Chakraborty S, Ramamurthy T, Nishibuchi M, YAMASAKI S, TAKEDA Y ET AL. Molecular 
evidence of clonal Vibrio parahaemolyticus pandemic strains. Emerg Infect Dis 2000; 6: 631-6.

35. Islam MS, Tasmin $R$, Islam Kahn $S$, Mahmud BaKht HB, Mahmood ZH, Ziaur Rahman M et al. Pandemic strains of 03:K6 Vibrio parahaemolyticus in the aquatic environment of Bangladesh. Can J Microbiol 2004; 50: 827-34.

36. Tuyet DT, Thiem VD, Von Seidiein L, Chowdhury A, Park E, CAHn DG ET AL. Clinical, epidemiological, and socioeconomic analysis of an outbreak of Vibrio parahaemolyticus in Khanh Hoa Province, Vietnam. J Infect Dis 2002; 186: 1615-20.

37. Park KS, Ono T, Rokuda M, Jang MH, Lda T, Honda T. Cytotoxicity and enterotoxicity of the thermostable direct hemolysin-deletion mutants of Vibrio parahaemolyticus. Microbiol Immunol 2004; 48: 313-8.

38. Tanabe T, Funahashi T, Nakao H, Miyoshi SI, Shinoda S, Yamamoto S. Identification and characterization of genes required for biosynthesis and transport of the siderophore vibrioferrin in Vibrio parahaemolyticus. J Bacteriol 2003; 185: 6938-49.

39. ButT AA, Aidridge KE, SAnders CV. Infections related to the ingestion of seafood Part I: Viral and bacterial infections. Lancet Infect Dis 2004; 4: 201-12.

40. US Food and Drug Administration. Vibrio, In CFSAN (ed.), Bacteriological Analytical Manual Online May 2004. USFDA 2004, 1-26.

41. Gooch JA, De Paola A, Kaysner CA, Marshall DL. Evaluation of two direct plating methods using nonradioactive probes for enumeration of Vibrio parahaemolyticus in oysters. Appl Environ Microbiol 2001; 67: 721-4.

42. Shapiro RL, Altekruse S, Hutwagner L, Bishop R, Hammond R, WiLson $S$ et al. The role of Gulf Coast oyster harvested in warmer months in Vibrio vulnificus infections in the United States, 19881996. J Infect Dis 1998; 178: 752-9.

43. HaLegraEFF GM. A review of harmful algal blooms and their apparent global increase. Phycologia 1993; 32: 79-99.

44. MorRIS JG, JR. Harmful algal blooms: An emerging public health problem with possible links to human stress on the environment. Annu Rev Energy Environ 1999; 24: 367-90.

45. Van Dolah FM. Marine algal toxins: origins, health effects, and their increased occurrence. Environ Health Perspect 2000; 108 Suppl 1: 133-41.
46. Dinubile MJ, Hoкama Y. The ciguatera poisoning syndrome from farm-raised salmon. Ann Intern Med 1995; 122: 113-4.

47. Fieming LE, Hiens Marine and Freshwater Biomedical Science Center. Paralytic shellfish poisoning. http:/ /www.whoi.edu/redtide/illness/psp.html.

48. The Harmful Algae Page. http://www.whoi.edu/ redtide/.

49. García C, Truan D, Lagos N. Veneno diameico de moluscos y derivados del bensopireno en bivalvos ahumados. Rev Chil Salud Pública 2004; 8: 162-9.

50. Fujski $H$, Suganuma $N$, Saguri $H$, Yoshizawa $S$, TAKagi K, NaKayasu M et al. New tumor promoters from marine natural products. American Chemical Society 1999: 232-9.

51. HaQ SM, Dayal HH. Chronic liver disease and consumption of raw oysters: A potentially lethal combination - A Review of Vibrio vulnificus septicemia. Am J Gastroenterol 2005; 100: 1195-9.

52. EPSTEIN PR. Emerging diseases and ecosystem instability: new threats to public health, 1995.

53. Fenical W, Committee on The Ocean's Role in Human Health, Commission on Geosciences Environment and RESOURCES. From monsoons to microbes. Understanding the ocean's Role in Human Health, National Academy Press, 2101 Constitution Ave, NW, Washington, DC 20418, 1999; 1-111.

54. SoLow AR. The coastal ocean is suffering from an overload of nutrients. Oceanus Mag (Red Tides and Dean Zones) 2004; 43: 43-5.

55. Reguera B. Capítulo 1: Establecimiento de un programa de seguimiento de microalgas tóxicas, In EA Sar, ME Ferrario, B Reguera (eds), Instituto Español de Oceanografía, 2002. Instituto Español de Oceanográfico de Vigo, Vigo 2002; 19-54.

Agradecimientos

Felipe C. Cabello agradece a la John Simon Guggenheim Memorial Foundation el otorgamiento de una Beca USA-Canadá 2004, que le permitiera viajar a Chile y la hospitalidad del Director del Instituto I-Mar de la Universidad de Los Lagos, Puerto Montt, Alejandro Buschman Ry las discusiones con él y con Daniel Varela Z, acerca de diversas materias de biología marina. Romilio Espejo T, agradece el apoyo del proyecto FONDECYT 1040805. Se agradece a las Sras. Betty Barría P y Harriett V Harrison la ayuda en la preparación del manuscrito. 